Note: This is an uncorrected version of an author's manuscript accepted for publication. Copyediting, typesetting, and review of the resulting proofs will be undertaken on this manuscript before final publication. During production and pre-press, errors may be discovered that could affect the content.

\title{
Identification of emotions in mixed disgusted-happy faces as a function of depressive symptom severity
}

\author{
Alvaro Sanchez $^{1 *}$, Nuria Romero ${ }^{1}$, Pierre Maurage $^{2}$, and Rudi De Raedt ${ }^{1}$ \\ 1. Ghent University, Belgium \\ 2. Université Catholique de Louvain, Belgium
}

\section{* Corresponding author:}

\author{
Alvaro Sanchez \\ Ghent University \\ Department of Experimental Clinical and Health Psychology \\ Henri Dunantlaan 2 \\ B-9000 Ghent \\ Belgium \\ Tel: +0032092649105 \\ E-mail: alvaro.sanchezlopez@ugent.be
}




\begin{abstract}
Background and Objectives: Interpersonal difficulties are common in depression, but their underlying mechanisms are not yet fully understood. The role of depression in the identification of mixed emotional signals with a direct interpersonal value remains unclear. The present study aimed to clarify this question. Methods: A sample of 39 individuals reporting a broad range of depression levels completed an emotion identification task where they viewed faces expressing three emotional categories (100\% disgusted and 100\% happy faces, as well as their morphed $50 \%$ disgusted - 50\% happy exemplars). Participants were asked to identify the corresponding depicted emotion as "clearly disgusted", "mixed", or "clearly happy". Results: Higher depression levels were associated with lower identification of positive emotions in $50 \%$ disgusted - 50\% happy faces. Limitations: The study was conducted with an analogue sample reporting individual differences in subclinical depression levels. Further research must replicate these findings in a clinical sample and clarify whether differential emotional identification patterns emerge in depression for different mixed negative-positive emotions (sad-happy vs. disgusted-happy). Conclusions: Depression may account for a lower bias to perceive positive states when ambiguous states from others include subtle signals of social threat (i.e., disgust), leading to an under-perception of positive social signals.
\end{abstract}

Keywords: Depression; cognitive biases; emotional identification; interpersonal functioning; mixed emotions 


\section{Introduction}

Depression is characterized by the experience of difficulties in social interactions (Gotlib \& Hammen, 2002). Depressed individuals tend to perceive social interactions as negative and to attribute the negative outcomes derived from these interactions to themselves (Beck, Rush, Shaw, \& Emery, 1979; Joiner \& Coyne, 1999). Such interpersonal dysfunctions may contribute to both the development and maintenance of depression (Rudolph, 2009), being associated with sustained negative affect and post-event rumination on negative outcomes (e.g., Kashdan \& Roberts, 2007). Current research is aimed to understand the mechanisms that may underlie such interpersonal difficulties. Cognitive models of depression (e.g., Beck, 2008) posit that information processing biases for positive and negative information play a critical role in the disorder, and may underlie interpersonal difficulties experienced by depressed individuals (Gotlib \& Hammen, 1992).

Supporting this assumption, depressed compared to healthy individuals are characterized by a reduced attention processing of positive social signals, such as others' happy faces (Armstrong \& Olatunji, 2012), and conversely by an increased attention processing of negative social signals, such as others' sad and disgusted faces (Armstrong \& Olatunji, 2012; Sanchez, Vazquez, Marker, LeMoult, \& Joormann, 2013; Sanchez, Romero \& De Raedt, 2017). These attentional biases have been shown to contribute to inefficient behavioral and emotional regulation during social interactions (Sanchez et al., 2017).

Furthermore, the way facial signals of emotion are identified and interpreted may also determine the production and regulation of affective states and behaviors in response to them (Phillips, Drevets, Rauch, \& Lane, 2003). Whereas an adequate identification of emotions in facial expressions provides crucial information to adequately adjust behavior to the interpersonal environment, biased facial emotion identification may lead to detrimental interpersonal 
interactions (Carton, Kessler, \& Pape, 1999). Therefore, it has been proposed that not only biased attention processing but also misinterpretation of emotional facial signals might be underlying interpersonal impairments in depression (Geerts \& Bouhuys, 1998). Growing research has started to examine the link between the ability to identify emotional expressions and depression. In standard experiments, pictures of real actors or schematic faces depicting different prototypical emotions are shown, and participants have to categorize the emotion depicted and/or judge its intensity. Results using this approach have been mixed (for a recent meta-analysis, see Dalili, Penton-Voak, Harmer, \& Munafo, 2015), with some studies supporting a depression-related impaired ability to identify prototypical facial expressions of emotions (e.g., Gur et al., 1992; Persad \& Polivy, 1993), but others failing to replicate those findings (e.g., Archer et al., 1992; Frewen \& Dozois 2005).

Given these inconsistent results, it has been argued that depressed individuals might be characterized by an accurate identification of prototypical high-intensity emotional expressions but show biases in labeling facial expressions when emotional states are more ambiguous and less prototypical (e.g., Beevers, Wells, Ellis, \& Fischer, 2010; Joormann \& Gotlib, 2006). Clarifying this question seems essential, as individuals most usually encounter subtle rather than fully intense or clearly prototypical emotional expressions in everyday social interactions. Initial research began testing this question by presenting both unambiguous (clearly expressed negative or clearly expressed positive emotions) and ambiguous (mixed similar amount of expressed negative-positive emotions) schematic faces, and asking participants to rate how strongly each schematic depicted positive and/or negative emotions. Results showed that depression was associated with rating of mixed negative-positive schematic pictures as more negative (Bouhuys et al., 1999; Geerts \& Bouhuys, 1998; Hale, 1998), and that such bias was associated with higher 
rumination levels (Raes, Hermans, \& Williams, 2006) and predicted persistence of depression across time (Bouhuys et al., 1999; Geerts \& Bouhuys, 1998).

Although these initial findings points to a depression-related negative bias in the identification of emotions in mixed negative-positive faces, results relied on the use of simple, schematic drawing of facial expressions. Further research requires using more ecologically valid stimuli (human facial expressions) varying in their degree in the negative-positive continuum. Beevers et al. (2010) presented a series of sad and fearful human faces morphed with happy faces of the same actors to varying degrees in the negative-positive continuum to dysphoric and nondysphoric individuals. Consistent with the assumption of an unimpaired prototypical emotional identification, groups did not differ in the identification of clearly prototypical expressions of those emotions. However, depression-related differences emerged in the identification of facial expressions depicting mixed negative-positive emotions. First, dysphoric individuals were more likely to identify sadness when viewing mixed sad-happy expressions. In contrast, dysphoric individuals were less likely to identify happiness in mixed fear-happy expressions. These results indicate that depression-related differences in the identification of mixed negative-positive facial expressions may vary depending on the nature of the negative emotion depicted, with more negative labeling when mixed faces depict mood-congruent negative emotions (sadness) but less positive labeling when they depict signals of physical threat (fear). However, both fearful and sad faces signal internal negative states within other persons. It seems likely that facial signals conveying negative relational information (e.g., personal rejection from others, as opposite to personal acceptance) may be highly relevant to depressed individuals (Gilboa-Schechtman, BenArtzi, Jeczemien, Marom, \& Hermesh, 2004).

In the present study, we examined depression-related processes of emotional identification in the negative-positive continuum using disgusted (i.e., rejecting) and happy (i.e., 
accepting) faces. We focused on the emotion of disgust, as current research points out a great perception of social threat for disgust faces (e.g., Amir et al., 2005). Furthermore, recent findings indicate a depression-related bias in attention processing of disgusted over happy faces during the confrontation of social interactions (Sanchez et al., 2017). A sample of individuals reporting a broad range of depression severity levels performed a task where they had to rate the emotions depicted in pictures representing clearly prototypical facial expressions of disgust and happiness, as well as in pictures representing morphed facial expressions of both emotions. We focused on $50 \%$ disgust $-50 \%$ happy morphed faces, as they represent the most ambiguous degree between both emotions in the negative-positive continuum.

Different to Beevers et al. (2010), who used a two-label forced-choice paradigm (i.e., indicate whether the facial expression is negative or positive), a three-label paradigm of response was employed, including an intermediate category (i.e., indicate whether the facial expression is 1) clearly negative, 2) mixed: not clearly negative nor clearly positive, or 3) clearly positive). Whereas a two-label paradigm may be adequate to index the emotional categorization of prototypical emotional expressions, it complicates to adequately index the perception of different levels of negative and positive emotions in mixed expressions. Kellough and Knight (2012) have shown that by asking participants to freely assign both positive and negative labels to propotypical and mixed negative-positive emotional faces, mixed expressions are identified as more negative than prototypical positive expressions and as more positive than prototypical negative expressions. Therefore, by using a three-label paradigm we were able to use a more ecologically valid procedure of emotional categorization that allowed to separately testing two different aspects of emotional identification. First, by using a forced-choice paradigm, we were able to test participants' accuracy in the labeling of prototypical picture categories (clearly 
negative and clearly positive expressions) to its corresponding emotion label (i.e., "clearly negative", "clearly positive", respectively). This served to test the hypothesis of an unimpaired identification of prototypical emotions in depression (Beevers et al., 2010). Second, by introducing a three-label paradigm we were able to analyze the probability of identifying different emotion labels in mixed emotional expressions (i.e., probability of identifying mixed expressions as clearly disgusted or as clearly happy, instead of as mixed). This enabled to analyze whether depression is associated with 1) a higher identification of disgust or 2) with a lower identification of happiness in mixed disgusted-happy expressions, as documented for other emotions in the negative-positive continuum (sad-happy, fear-happy faces, respectively; Beevers et al., 2010).

\section{Material and Methods}

\subsection{Participants}

Participants with minimal to severe depressive symptoms were sampled from the Ghent University research participant pool based on a prescreening measure (Mood and Anxiety Symptom Questionnaire; Clark \& Watson, 1991). At testing, 39 individuals (36 female; age range: 18-36) reported a broad range of depressive symptom severity levels (range: $0-42, M=11.28$, $S D=10.50$ ) on the Beck Depression Inventory-II (Beck, Steer, \& Brown, 1996; Dutch translation: Van der Does, 2002), with 25 individuals reporting minimal (20: 0-9; 5: 10-13), 7 mild (14-19), 4

moderate (20-28), and 3 severe symptom levels (29-63). All participants were native Dutch speakers with normal or corrected-to-normal vision. They provided informed consent and were paid 15 euro. The study was approved by the faculty ethical review board at Ghent University. 


\subsection{Depressive Symptom Severity}

The BDI-II assessed depressive symptom severity. On 21 items rated on a four-point scale, respondents indicated the extent to which they suffered from depressive symptoms in the past two weeks. This measure has good reliability and validity in both healthy and depressed samples (Beck et al., 1996; Van der Does, 2002). The internal consistency in this study was $\alpha=.95 .{ }^{1}$

\subsection{Emotion Identification Task}

\subsubsection{Stimuli}

Stimuli comprised 8 computer-generated facial models (4 male and 4 female models) created with FaceGen Modeller 3.9. (Singular Inversions, Toronto, ON, Canada), which allows to build and modulate realistic emotional facial expressions on the basis of the FACS system (Ekman \& Friesen, 1976). Two faces were then first created for each model, respectively expressing full-blown emotions of happiness and disgust. Then, a morphing procedure was used to mix the two emotional faces in order to obtain mixed emotional expressions (50\% happiness 50\% disgust), leading to three faces (100\% disgusted, 50\%-50\% mixed, 100\% happy) per model

(see Figure 1) and a total of 24 stimuli. All stimuli were then standardized using Photoshop 15.1: they were placed on a black background, resized to a $400 \times 400$ pixels format with a resolution of $96 \mathrm{ppm}$ (stimuli subtended a visual angle of $8 \times 4$ degrees).

\footnotetext{
${ }^{1}$ Given that high levels of general anxiety and social anxiety are common among individuals with high depressive symptom severity (e.g., Kessler et al., 1996), the study also included the control of anxiety measures as covariates. Further evaluation included self-reported measures of trait and state anxiety, with the Trait-State Anxiety Inventory (STAI, Spielberger et al., 1970), as well as of fear of negative evaluation (a common symptom in social anxiety), with the Fear of Negative Evaluation scale (FNE: Watson and Friend, 1969). Trait and state anxiety as well as fear of negative evaluation were highly correlated with depressive symptom severity in our study: $r=.85, p=.001 ; r=.77$, $p=.001 ; r=.42, p=.008$, respectively. Further analyses were conducted including anxiety measures as covariates in the main analyses (see footnote 2), as well as partial correlations controlling for anxiety measures, in order to replicate the findings obtained in the main analyses (see footnote 3 ).
} 


\subsubsection{Procedure}

Pictures were successively shown on a computer screen and participants were instructed to identify the emotion expressed by the face, by indicating whether they perceived it as "clearly disgusted", "mixed: not clearly disgusted nor clearly happy", or "clearly happy". Each trial began with a central fixation cross presented for $500 \mathrm{~ms}$. The picture $(5.8 \times 7.5 \mathrm{~cm})$ was then presented on the screen along with the three labels: 1 - disgusted, 2 - not disgusted nor happy, 3 - happy. Participants then selected which label best described the emotion expressed in the picture by pressing the appropriate key. Once participants rated the picture, the next trial began. The rating task comprised a total of 24 trials. Images were presented in a different randomized order for each participant.

Figure 1. Example of model representing the three possible picture emotion categories.

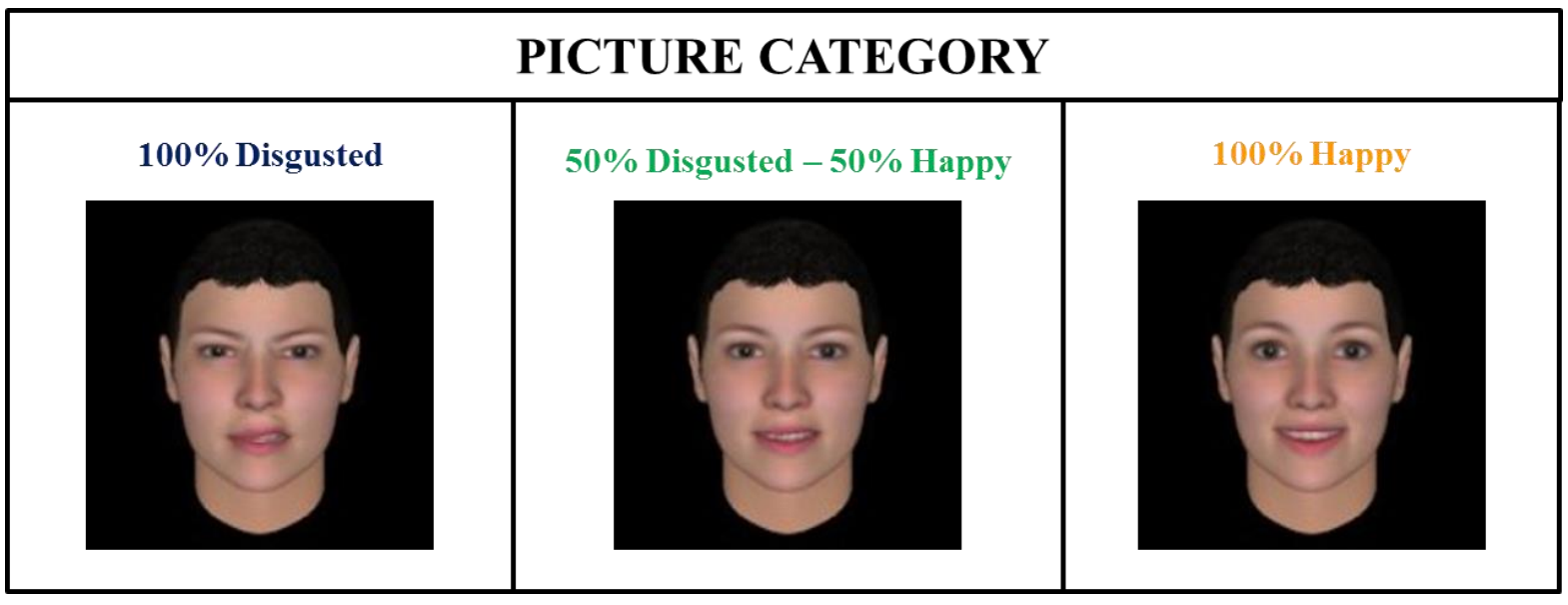

\subsection{Statistical approach}

The data-analytic strategy comprised the evaluation of the role of depressive symptom severity in the level of emotional identification across the disgust-happiness continuum for each 
emotional picture category (i.e., 100\% disgusted, 50\%-50\% mixed and 100\% happy expressions). Emotional identification indices were computed based on Beevers et al. (2010): First, cases labeled as "clearly disgusted" were scored as 0 , cases labeled as "mixed" were scored as 0.5 , and cases labeled as "clearly happy were scored as 1 . Then, the emotional identification index for each type of emotional picture category was derived by averaging their scores, with the resulting indices varying within a range from 0 (identification of all expressions in that emotional category as "clearly negative") to 1 (identification of all expressions in that emotional category as "clearly positive"), allowing to test participants' accuracy in the labeling of prototypical picture categories. Whereas Beevers et al. (2010) used this formula to compute emotional identification biases using a two-forced choice paradigm (e.g., indicate whether expressions were identified as "negative" $=$ score 0 , or as "positive" = score 1 , and then compute the average of those scores for each emotional category), their two-label approach did not allow to account for intermediate labels (i.e., identification of mixed negative-positive emotions in the expressions), being less suitable to interpret results in the identification of mixed negative-positive emotional categories. The inclusion of a third label in our study ("mixed") allowed to derive a more precise estimation of biases in the emotional identification of mixed expressions, with scores around 0.5 indicating identification of the expressions in that category as mixed, and scores bellow and above 0.5 indicating biases to perceive more negative and positive emotions in the mixed expressions, respectively.

To increase the statistical power in our analyses, we first conducted a repeated measures analysis of covariance (ANCOVA) of the emotional identification indices, with emotional category ("clearly disgusted", " mixed", "clearly happy" pictures) as within-subject factor and depressive symptom severity level as covariate. This analysis served to examine the main 
differences in emotional identification between emotional categories across the whole sample as well as the depression severity level by emotional picture category interaction . P-values were Greenhouse-Geisser corrected to address violation of the assumption of sphericity. When there was a significant interaction between depressive symptom severity and emotional category in the ANCOVA, the interaction was decomposed via separate correlations tests for each type of emotional picture category. Bivariate Pearson correlations were performed, separately testing the hypothesis of an unimpaired identification of prototypical emotions in depression (research question 1: correlations between depressive symptom severity and emotional identification levels for clearly disgusted and clearly happy expressions), and whether depression is associated with higher identification of disgust / lower identification of happiness in mixed disgusted-happy expressions (research question 2: correlations between depressive symptom severity and emotional identification levels for mixed expressions).

\section{Results}

Mean and standard deviations for the emotional identification indices of each picture emotional category are shown in Table 1.

Table 1. Mean and standard deviations of emotional identification for each picture category in the total sample

\begin{tabular}{ccc}
\hline \multicolumn{3}{c}{ Picture Category } \\
\cline { 1 - 2 }$\frac{100 \% \text { disgusted }}{M(S D)}$ & $\frac{50 \% \text { disgusted }-50 \% \text { happy }}{M(S D)}$ & $100 \%$ happy \\
\hline $0.05(0.08)$ & $0.70(0.15)$ & $M(S D)$ \\
\hline
\end{tabular}

Note. $M=$ Mean; $S D=$ Standard deviation 
The ANCOVA, with picture emotional category (100\% disgusted, 50\% disgusted $-50 \%$ happy, and 100\% happy pictures) and depressive symptom severity as covariate, showed a significant main effect of picture emotional category, $F(1.62,60.11)=474.06, p=.001, \eta_{p}{ }^{2}=$ .93. Post-hoc comparisons showed that $100 \%$ disgusted expressions were labeled as more negative than both $50 \%$ disgusted - 50\% happy and 100\% happy pictures, and 100\% happy pictures were labeled as more positive than $50 \%$ disgusted $-50 \%$ happy pictures, all $p$ 's $=.001$. Further paired-samples t-tests of each emotional identification index against a value of 0.5 (i.e., indicative of identification of mixed/similar level of negative and positive emotions) showed that participants rated $100 \%$ disgusted pictures as "negative" (i.e., significantly below the 0.5 criteria, $\mathrm{t}(38)=-33.19, p=.001$ ) and $100 \%$ happy pictures as "positive" (i.e., significantly above the 0.5 criteria, $\mathrm{t}(38)=61.70, p=.001)$. Furthermore, although at a lower level than for 100\% happy pictures, participants also rated 50\% disgusted $-50 \%$ happy pictures as positive (i.e., significantly above the 0.5 criteria, $\mathrm{t}(38)=8.65, p=.001)$, indicating a positive bias to perceive a higher level of positive than negative emotions in mixed expressions.

Furthermore, the main effect of the depressive symptom severity covariate in the ANCOVA was found to reach significance, $F(1,37)=6.64, p=.014, \eta_{p}^{2}=.15$. Importantly, there was also a significant depressive symptom severity by picture emotional category interaction., $F(1.62,60.11)=4.64, p=.013, \eta_{p}{ }^{2}=.11 .^{2}$ To further investigate this interaction effect, bivariate correlation coefficients between depressive symptom severity and emotional identification indices for each picture emotional category were calculated. First, regarding to the

\footnotetext{
${ }^{2}$ Further ANCOVAs were conducted introducing trait and state anxiety and their interaction, as well as fear of negative evaluation as covariates in the model. Different to depressive symptom level, neither trait and state anxiety nor their interaction accounted for main effects in picture emotional category, all $F$ 's $<1.61$, all $p$ 's $>.20$, all $\eta_{p}{ }^{2}<$ .05. Similarly, fear of negative evaluation did not significantly interacted with picture emotional category, $F(1.62,60.11)=1.69, p=.19, \eta_{p}^{2}=.04$.
} 
role of depression in emotional identification of prototypical expressions (research question 1), analyses showed that higher depression scores were not significantly associated with differential levels of emotional identification of $100 \%$ disgusted pictures, $r=-.27, p=.10$, or $100 \%$ happy pictures, $r=.09, p=.60$. Therefore, the hypothesis of an unimpaired identification of prototypical emotional expressions in depression was supported both for expressions of disgust and happiness. Second, regarding to the role of depression in the emotional identification of mixed disgustedhappy faces (research question 2), higher depression scores were significantly associated with lower scores in the emotional identification of $50 \%$ disgusted $-50 \%$ happy pictures, $r=-.40, p=$ $.01^{3}$

Overall, results indicated a lower positive bias in the identification of mixed expressions as happy at higher depression levels ${ }^{4}$. Differential levels of $50 \%$ disgusted $-50 \%$ happy pictures' identification as a function of depressive symptom severity levels are depicted in Figure 2.

\footnotetext{
${ }^{3}$ Despite that anxiety measures did not accounted for differences in emotional identification of picture emotional categories, as observed in the further ANCOVAs, we aimed to replicate the main findings for depression severity levels after controlling for anxiety measures (i.e., trait and state anxiety, and fear of negative evaluation). A partial correlation controlling both for trait and state anxiety, indicated that the significant negative association between depression and emotional identification of 50\% disgusted $-50 \%$ happy pictures became marginally significant, $r=$ $.29, p=.08$. In contrast, after controlling for the social anxiety measure (fear to negative evaluations), higher depression scores remained significantly associated with lower levels of identification of 50\% disgusted - 50\% happy pictures as "happy", $r=-.35, p=.03$.

${ }^{4}$ The distribution of BDI-II scores in our sample, by design, did not meet the assumption of normality, $\mathrm{S}-\mathrm{W}=.86$, $\mathrm{df}=39, p=.001$. The distribution of BDI-II scores showed a positive skewness $=1.36, \mathrm{SE}=0.38$, and kurtosis $=1.56$, $\mathrm{SE}=0.74$. In order to assure that our main findings were not affected by the skewed distribution of depression symptom severity levels in the sample, BDI-II scores were square root transformed to meet the assumption of normality, $\mathrm{S}-\mathrm{W}=.98, \mathrm{df}=39, p=.55$, skewness $=-0.23, \mathrm{SE}=0.38$, kurtosis $=-0.63, \mathrm{SE}=0.74$, and analyses were repeated introducing transformed BDI-II scores as covariate. Further analyses showed that the depressive symptom severity by picture emotional category interaction in the ANCOVA when using transformed BDI-II scores as covariate remained significant, $F(1.62,60.14)=3.44, p=.04, \eta_{\mathrm{p}}{ }^{2}=.09$. Further correlation analyses confirmed no associations between transformed depression scores and emotional identification of prototypical disgusted and happy expressions, $r=-.24, p=.15$, and $r=.07, p=.69$, respectively, and a significant association between higher transformed depression scores and lower identification of happiness in mixed expressions, $r=-.35, p=.03$.
} 
Figure 2. Distribution of the proportions of emotional identification of each picture emotional category as a function of depressive symptom severity.

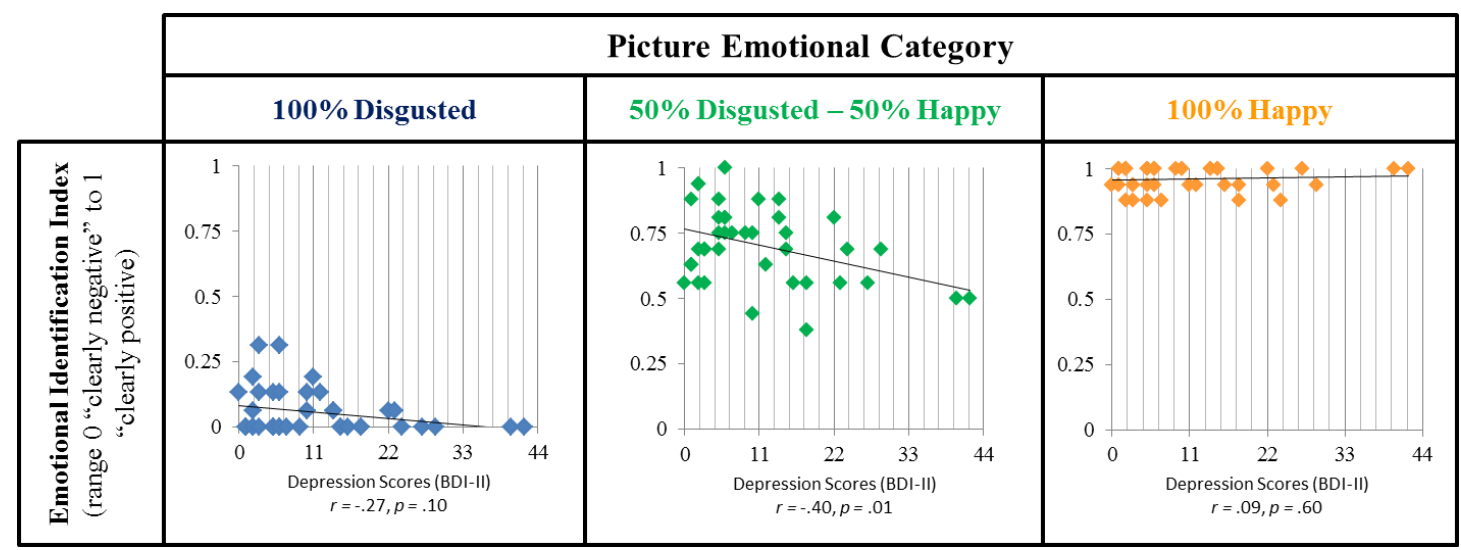

Notes. Pearson correlation coefficients $* p<.05 ; * * p<.01$

\section{Discussion and Conclusions}

The present study aimed to clarify the role of depression in the identification of emotional signals with a direct interpersonal value (disgusted-rejecting as opposite to happy-accepting faces), thus offering a direct exploration of social interaction biases in depression.

The hypothesis of an unimpaired identification of prototypical emotions in depression (Beevers et al., 2010) was confirmed both for clearly disgusted and happy expressions. Consistently with previous findings (e.g., Beevers et al., 2010; Joormann \& Gotlib, 2006), participants in our study demonstrated an accurate identification of prototypical expressions, not affected by differences in depressive symptom severity levels. In contrast, depression level specifically accounted for differences in labeling facial expressions when emotional states were ambiguous (mixed disgusted-happy facial expressions). Our results showed a positive bias to identify mixed disgusted-happy facial expressions as "clearly positive" in the sample, with this bias being significantly lower at higher depression levels (see Figure 2). Therefore, with regard to the question on whether depression is associated with 1) a higher identification of disgust and/or 
2) with a lower identification of happiness in mixed disgusted-happy expressions, the latter assumption was supported. Our results contrast with previous studies using schematic faces, where it has been shown a negative bias in depression consisting on a higher identification of negative emotions in mixed negative-positive expressions (Bouhuys et al., 1999; Geerts \& Bouhuys, 1998; Hale, 1998). However, using more ecologically valid stimuli (human facial expressions), Beevers et al. (2010) have demonstrated a similar depression-related negative bias of higher identification of negative emotions in mixed expressions conveying mood-congruent states (i.e., sadness), but a different pattern consisting on lower identification of positive emotions when mixed expressions conveyed signals of physical threat (i.e. fear). Consistently, in our study a lower identification of happiness in mixed expression conveying social threat signals (i.e., disgust) was associated with higher depression levels. Taken together, these results might be indicative of differential processes of emotional identification in mixed emotions as a function of the nature of negative signals in the environment: Whereas depression might be associated with a negative bias to selectively perceive mood-congruent negative states in ambiguous or mixed emotional states from others (i.e., sadness), they might lack a positive bias to selectively perceive positive states when ambiguous or mixed states from others include signals of threat (i.e., disgust).

On one hand, depressed individuals may be characterized by a biased identification of subtle negative emotions in others that are congruent with their mood state (i.e., sadness; Beevers et al., 2010). Joormann and Gotlib (2006) explored this possibility by using an innovative design in which participants were presented with a sequence of faces that expressed increasing degrees of emotional intensity, slowly changing from a neutral to a full-intensity sad, angry or happy expression. Participants were instructed to stop the sequence when they could identify an 
emotion. Interestingly, depressed individuals required less intensity to identify sadness in comparison to anger in others' facial expressions, indicating a readiness to perceive moodcongruent negative states over other types of negative states in others. These results are congruent with Beck's (1976) prediction of content specificity, by which a negative bias in depression would occur for information congruent with depressed individuals' mood state.

On the other hand, our results suggest that, in absence of negative mood-congruent information (i.e., sadness) depression would be associated with a lower perception of positive emotions from others when confronted with subtle or mixed negative social information (i.e., disgust). In the context of social threat, such lower positive bias in the identification of mixed emotions might account for differential processes of interpretation of social interactions. Previous research has shown that healthy individuals are characterized by a positive inferential bias to favor positive interpretations of ambiguous social situations (see, for instance, Hirsch \& Mathews, 1997, 2000). In contrast, these studies have also shown that individuals characterized by social interaction impairments (in the context of social anxiety) were characterized by a balanced generation of threatening and positive interpretations (i.e., absence of healthy individuals' positive bias) rather than by a negative bias favoring threatening interpretations of ambiguous situations. This evidence has been extended to the context of depression by Moser, Huppert, Foa, and Simmons (2012), who tested interpretation biases for socially ambiguous scenarios, separately in individuals meeting diagnostic criteria for social phobia, major depression disorder/dysthymia, and both comorbid social phobia and depression diagnoses, in comparison to healthy controls. In this study, healthy controls showed a positive interpretation bias both at a behavioral level (i.e., faster reaction times to unsolved ambiguity into a positive vs. a negative interpretation) and a neural level (i.e., event-related potentials, ERP: enhanced N4 
amplitudes for negative than for positive solutions of the social ambiguity). However, the three clinical groups, including clinically depressed individuals without social phobia comorbidity, were characterized by absence of the 'healthy' positive interpretation bias, as observed both in their reaction times and their ERPs when solving social ambiguity. Since real social interactions rarely provide clear evidence of rejection or acceptance from others, such ultimate interpretation and its impact in emotional response might be dependent on individuals' perception of social signals in the environment. The positive bias to perceive mixed disgusted-happy expressions as clearly happy observed at lower depression levels in our study might, in turn, facilitate a biased positive interpretation of ambiguous social environments in healthy individuals, as suggested by Hirsch and Mathews $(1997,2000)$ and Moser et al. (2012). A higher propensity to generate more positive interpretations of ambiguous scenarios would act as an adaptive mechanism facilitating resilience against stress and protecting from depression (e.g., Kleim, Thörn, \& Ehlert, 2014). In contrast, the lower perception of positive emotions in mixed rejecting-accepting social signals observed at higher levels of depression in our study might lead to a more balanced interpretation of socially ambiguous scenarios, as observed both in depression and other problems reflecting social impairments (Hirsch and Mathews, 1997, 2000; Moser et al. (2012). Of note, consistent with the lack of positive interpretation biases for social ambiguous information in depression even in absence of social anxiety comorbidity (Moser et al., 2012), further analyses in or study confirmed the evidence for an association between depression and a lower positive identification bias for mixed/ambiguous expressions, even after statistically controlling for co-varying social anxiety symptoms (i.e., fear to negative evaluations). Taking these results together, a possible explanation on why depressed individuals may end up attributing to themselves more negative social performance and more negative outcomes from balanced social contexts, might be that in absence of protective positive biases for external social information, they ignore external social 
signals and rely instead on internal sources of information (i.e., negative beliefs about oneself, independently of perceived-interpreted external evidence in the social environment) to form their social judgments. This open question require further research to clarify the interplays between socio-emotional identification and interpretation processes and their role in social performance and post-social events evaluation.

However, further research should first clarify the differential emotional identification patterns reported for different mixed negative-positive emotions by Beevers et al. (2010) and the present study (i.e., negative bias for mixed sad-happy faces, absence of positive bias for mixed disgusted-happy faces). A limitation in our study is that we only examined the disgust-happiness continuum and, therefore, it cannot be determined whether the results found using our methodology would reflect a social-specific bias or are part of a broader, more generalized lack of positive bias in the emotional identification of mixed negative-positive facial expressions in depression. Of particular relevance, it remains to be tested whether an opposite depression-related negative bias in the sadness-happiness continuum would also be observed using our methodological approach. Whether depression may be characterized by a biased identification of sadness in mixed sad-happy faces (in contrast to a non-biased identification of happiness in mixed disgusted-happy faces) will require methods allowing indexing multiple levels of categorical perception, as done in our study. Another limitation is that most of the participants in our sample were women, which may limit the generalizability of the results. Furthermore, the non-clinical nature of the recruited sample also limits to some extent the generalizability of our findings. Further investigation is needed across different samples representing the depression course, including clinically depressed individuals, in order to clarify whether lower identification of happiness in mixed disgusted-happy expressions is replicated in clinical depression and the 
implications of this lack of positive bias. Whereas several studies have proven a bias in clinically depressed individuals to easily detect sadness in mixed sad-neutral expressions (see Dalili et al., 2015), the experience/lack of biases in the identification of mixed positive-negative social signals remains to be tested. Finally, whereas our study and previous research have focused in evaluating the emotional identification of single individually presented prototypical or mixed emotional expressions, the social environment usually involves interactions with multiple others' reflecting dynamically changing levels in their emotional states and their intensities. Further research must consider how emotional identification processes operate in depression in the context of more complex and ecologically valid social environments (both single and multiple sources of prototypical and mixed emotions in dynamic change).

In sum, the results in this study promise to increase the current knowledge on social interaction biases in depression. The methodology employed served to clarify the conditions under which emotional identification biases occurs during the processing of interpersonally relevant emotion signals (disgust/rejection-happiness/acceptance continuum). Our results suggest that depression may account for a lower positive bias to selectively perceive positive states when viewing mixed positive-negative interpersonal states from others. Such under-perception of positive emotional states in others' mixed expressions may have important implications for the evaluation of social signals and, therefore, for social functioning. 


\section{Acknowledgements, declaration of interest, and role of funding organizations}

This research was supported by a grant of the Research Foundation Flanders (FWO) awarded to the first author and a grant BOF16/GOA/017 for Concerted Research Actions of Ghent University awarded to the last author. The authors declare no conflict of interest. 


\section{References}

Amir, N., Klumpp, H., Elias, J., Bedwell, J. S., Yanasak, N., \& Miller, L. S. (2005). Increased activation of the anterior cingulate cortex during processing of disgust faces in individuals with social phobia. Biological Psychiatry, 57(9), 975-981. http://doi.org/10.1016/j.biopsych.2005.01.044

Archer, J., Hay, D. C., \& Young, A.W. (1992). Face processing in psychiatric conditions. British Journal of Clinical Psychology, 31, 45-61. http://doi.org/10.1111/j.20448260.1992.tb00967.x

Armstrong, T., \& Olatunji, B. O. (2012). Eye tracking of attention in the affective disorders: A meta-analytic review and synthesis. Clinical Psychology Review, 32(8), 704-723. http://doi.org/10.1016/j.cpr.2012.09.004

Beck, A. T. (1976). Cognitive therapy and the emotional disorders. Oxford, England: International Universities Press.

Beck, A. T. (2008). The evolution of the cognitive model of depression and its neurobiological correlates. American Journal of Psychiatry, 165(8) 969-977. http://doi.org/10.1176/appi.ajp.2008.08050721

Beck, A. T., Rush, J. A., Shaw, B. F., \& Emery, G. (1979). Cognitive therapy for depression. New York: Guilford Press

Beck, A. T., Steer, R. A., \& Brown, G. K. (1996). Manual for the Beck Depression Inventory - II. San Antonio, TX: Psychological Corporation.

Beevers, C. G., Wells, T. T., Ellis, A. J., \& Fischer, K. (2010). Identification of emotionally ambiguous interpersonal stimuli among dysphoric and nondysphoric individuals. Cognitive Therapy and Research, 33(3), 283-290. http://doi.org/10.1007/s10608-008-9198-6

Bouhuys, A. L., Geerts, E., \& Gordijn, M. C. (1999). Depressed patients' perceptions of facial emotions in depressed and remitted states are associated with relapse: a longitudinal study. Journal of Nervous and Mental Disease, 187(10), 595-602.

Carton, J. S., Kessler, E. A., \& Pape, C. L. (1999). Nonverbal decoding skills and relationship well-being in adults. Journal of Nonverbal Behavior, 23(1), 91-100. http://doi.org/10.1023/A:1021339410262

Clark, L. A., \& Watson, D. (1991). Tripartite model of anxiety and depression: psychometric evidence and taxonomic implications. Journal of Abnormal Psychology, 100(3), 316-36.

Dalili, M. N., Penton-Voak, I. S., Harmer, C. J., \& Munafo, M. R. (2015). Meta-analysis of emotion recognition deficits in major depressive disorder. Psychological Medicine, 45, 1135-1144. http://doi.org/ 10.1017/S0033291714002591

Ekman, P., \& Friesen, W. V. (1976). Pictures of Facial Affect. 1st ed. Palo Alto, CA: Consulting Psychologists Press. 
Frewen, P. A., \& Dozois, D. J. A. (2005). Recognition and interpretation of facial expressions in dysphoric women. Journal of Psychopathology and Behavioral Assessment, 27, 305-315. http://doi.org/10.1007/s10862-005-2410-z

Geerts, E., \& Bouhuys, N. (1998). Multi-level prediction of short-term outcome of depression: Non-verbal interpersonal processes, cognitions and personality traits. Psychiatry Research, 79(1), 59-72. http://doi.org/10.1016/S0165-1781(98)00021-3

Gilboa-Schechtman, E., Ben-Artzi, E., Jeczemien, P., Marom, S., \& Hermesh, H. (2004). Depression impairs the ability to ignore the emotional aspects of facial expressions: Evidence from the Garner task. Cognition and Emotion, 18, 209-231.

Gotlib, I. H., \& Hammen, C. L. (1992). Psychological aspects of depression: Toward a cognitiveinterpersonal integration. Oxford, England: John Wiley and Sons.

Gur, R. C., Erwin, R. J., Gur, R. E., Zwil, A. S., Heimberg, C., Kraemer, H. C. (1992). Facial emotion discrimination: II. Behavioral findings in depression. Psychiatry Research, 42, 241-251. http://doi.org/10.1016/0165-1781(92)90116-K

Hale, W. W. (1998). Judgment of facial expressions and depression persistence. Psychiatry Research, 80(3), 265-274. http://doi.org/10.1016/S0165-1781(98)00070-5

Hirsch, C., \& Mathews, A. (1997). Interpretative inferences when reading about emotional events. Behaviour Research and Therapy, 35(12), 1123-1132. http://doi.org/10.1016/S00057967(97)00069-7

Hirsch, C. R., \& Mathews, a. (2000). Impaired positive inferential bias in social phobia. Journal of Abnormal Psychology, 109(4), 705-712. http://doi.org/10.1037/0021-843X.109.4.705

Joiner, T., \& Coyne, J.C. (1999). Advances in interpersonal approaches: the interactional nature of depression. Washington DE: American Psychological Association.

Joormann, J., \& Gotlib, I. H. (2006). Is this happiness I see? Biases in the identification of emotional facial expressions in depression and social phobia. Journal of Abnormal Psychology, 115(4), 705-714. http://doi.org/10.1037/0021-843X.115.4.705

Kashdan, T. B., \& Roberts, J. E. (2007). Social anxiety, depressive symptoms, and post-event rumination: Affective consequences and social contextual influences. Journal of Anxiety Disorders, 21(3), 284-301. http://doi.org/10.1016/j.janxdis.2006.05.009

Kellough, J. L., \& Knight, B. G. (2012). Positivity Effects in Older Adults ' Perception of Facial Emotion: The Role of Future Time Perspective. The Journals of Gerontology, 67, 150-158. http://doi.org/10.1093/geronb/gbr079.

Kessler, R. C., Nelson, C. B., McGonagle, K. A., Liu, J., Swartz, M., \& Blazer, D. G. (1996). Comorbidity of DSM-III-R major depressive disorder in the general population: Results from the U.S. National Comorbidity Survey. The British Journal of Psychiatry, 168(30):821. 
Kleim, B., Thörn, H. A., \& Ehlert, U. (2014). Positive interpretation bias predicts well-being in medical interns. Frontiers in Psychology, 5(JUN), 1-6.

http://doi.org/10.3389/fpsyg.2014.00640

Moser, J. S., Huppert, J. D., Foa, E. B., \& Simons, R. F. (2012). Interpretation of ambiguous social scenarios in social phobia and depression: Evidence from event-related brain potentials. Biological Psychology, 89, 387-397. http://doi.org/ 10.1016/j.biopsycho.2011.12.001

Persad, S. M., \& Polivy, J. (1993). Differences between depressed and nondepressed individuals in the recognition of and response to facial cues. Journal of Abnormal Psychology, 102, 358-368. http://doi.org/10.1037/0021-843X.102.3.358.

Phillips, M. L., Drevets, W. C., Rauch, S. L., \& Lane, R. (2003). Neurobiology of emotion perception I: The neural basis of normal emotion perception. Biological Psychiatry, 54(5), 504-514. http://doi.org/10.1016/S0006-3223(03)00168-9

Raes, F., Hermans, D., \& Williams, J. M. G. (2006). Negative Bias in the Perception of Others' Facial Emotional Expressions in Major Depression. Journal of Nervous and Mental Disease, 194(10), 796-799. http://doi.org/10.1097/01.nmd.0000240187.80270.bb

Rudolph, K. D. (2009). The interpersonal context of adolescent depression. In S. NolenHoeksema, L. M. Hilt, S. Nolen-Hoeksema, \& L. M. Hilt (Eds.), Handbook of depression in adolescents (pp. 377-418). New York, NY: Routledge Taylor \& Francis

Sanchez, A., Romero, N., \& De Raedt, R. (2017). Depression-related difficulties disengaging from negative faces are associated with sustained attention to negative feedback during social evaluation and predict stress recovery. Manuscript under submission.

Sanchez, A., Vazquez, C., Marker, C., LeMoult, J., \& Joormann, J. (2013). Attentional disengagement predicts stress recovery in depression: an eye-tracking study. Journal of Abnormal Psychology, 122(2), 303-13. http://doi.org/10.1037/a0031529

Spielberger, C. D., Gorsuch, R. L., \& Lushene, R. E. (1970). State-trait anxiety inventory. Consulting Psychologists Press, Palo Alto.

Van der Does, A. J. W. (2002). Handleiding bij de Nederlandse versie van de Beck Depression Inventory - second edition (BDI-II-NL). [The Dutch version of the Beck Depresion Inventory-II]. Lisse, The Netherlands: Swets \& Zeitlinger.

Watson, D., \& Friend, R. (1969). Measurement of social-evaluative anxiety. Journal of Consulting and Clinical Psychology, 33(4), 448-457. doi: 10.1037/h0027806 